\title{
Imaging, biomarker and invasive assessment of diffuse left ventricular myocardial fibrosis in atrial fibrillation
}

\author{
Gordon A. Begg ${ }^{1,2^{*}}$ (D, Peter P. Swoboda ${ }^{2}$, Rashed Karim ${ }^{3}$, Tobias Oesterlein ${ }^{4}$, Kawal Rhode ${ }^{3}$, Arun V. Holden ${ }^{5}$, \\ John P. Greenwood ${ }^{1}$, Eduard Shantsila ${ }^{6}$, Gregory Y. H. Lip ${ }^{7,8}$, Sven Plein ${ }^{1}$ and Muzahir H. Tayebjee ${ }^{1}$
}

\begin{abstract}
Background: Using cardiovascular magnetic resonance imaging (CMR), it is possible to detect diffuse fibrosis of the left ventricle (LV) in patients with atrial fibrillation (AF), which may be independently associated with recurrence of AF after ablation. By conducting CMR, clinical, electrophysiology and biomarker assessment we planned to investigate LV myocardial fibrosis in patients undergoing AF ablation.

Methods: LV fibrosis was assessed by T1 mapping in 31 patients undergoing percutaneous ablation for AF. Galectin-3, coronary sinus type I collagen C terminal telopeptide (ICTP), and type III procollagen $N$ terminal peptide were measured with ELISA. Comparison was made between groups above and below the median for LV extracellular volume fraction (ECV), followed by regression analysis.
\end{abstract}

Results: On linear regression analysis LV ECV had significant associations with invasive left atrial pressure (Beta $0.49, P=$ 0.008) and coronary sinus ICTP (Beta $0.75, P<0.001$ ), which remained significant on multivariable regression.

Conclusion: LV fibrosis in patients with AF is associated with left atrial pressure and invasively measured levels of ICTP turnover biomarker.

Keywords: Atrial fibrillation, Cardiovascular magnetic resonance imaging, Fibrosis, Biomarkers, Voltage mapping

\section{Background}

Percutaneous pulmonary vein (PV) isolation is often used for rhythm control in patients with atrial fibrillation (AF). However, at least one third of such patients experience a recurrence of AF even after multiple procedures [1]. This is most commonly due to reconnection of the pulmonary veins, however in a significant proportion of patients this is not the case and the mechanism(s) in these instances is unclear. Identification of AF patients who are likely to maintain sinus rhythm after the procedure is important, to reduce unnecessary exposure to procedural risks and expense.

Fibrosis is a hallmark of the left atrial (LA) pathological changes associated with AF development and recurrence

\footnotetext{
* Correspondence: gordon.begg@nhs.net

'Department of Cardiology, Leeds General Infirmary, X39 Cardiology Offices, Great George St, Leeds LS1 3EX, UK

${ }^{2}$ Leeds Institute of Cardiovascular and Metabolic Medicine, University of Leeds, Clarendon Way, Leeds LS2 9JT, UK

Full list of author information is available at the end of the article
}

after ablation, and research has explored the clinical utility of LA fibrosis assessment by various methods [2-6].

However, left ventricular (LV) fibrosis is also more prominent in AF patients than those without AF, and may be a predictor of AF recurrence [7, 8]. Diffuse LV fibrosis can be estimated using cardiovascular magnetic resonance (CMR), by calculating the extracellular volume fraction (ECV) from native and post contrast T1 mapping [9].

Circulating biomarkers such as type I collagen $C$ terminal telopeptide (ICTP), type III procollagen $\mathrm{N}$ terminal peptide (PIIINP) and galectin 3 (gal-3) are markers of fibrosis that can be measured in the bloodstream [2]. They offer minimally invasive assessment of fibrosis, and would be a useful tool for improving patient selection if their clinical utility in doing so could be confirmed. They may also have a research application, in defining the mechanism of AF.

Although LV fibrosis has been associated to some extent with $\mathrm{AF}$ and $\mathrm{AF}$ recurrence after treatment, the mechanism behind this association is not clear. Raised 
LA pressure has been associated with recurrence of AF after catheter ablation, however the relationship between LA pressure and ventricular cardiac fibrosis in AF patients has not been studied in depth [10]. LA pressure is a routinely available direct measurement during AF procedures after trans-septal puncture, and further study may provide mechanistic insights into any haemodynamic influence on LV fibrosis in this patient group.

We investigated the interaction between LV fibrosis, LA fibrosis, and LA pressure, all of which have been associated with arrhythmia recurrence in patients after AF ablation. This interaction was examined in a multi-modality fashion, using CMR, invasive LA voltage mapping, LA pressure measurement and circulating biomarker assays. We hypothesized that LA fibrosis, measured by voltage mapping, is associated with diffuse LV fibrosis, measured by T1 mapping, and that raised LA pressure is associated with both of these measures. To attempt to gain a mechanistic insight into the pathological process of the fibrosis identified via these imaging methods, we also tested levels of circulating fibrosis biomarkers, including from intracardiac blood.

\section{Methods}

Ethical approval was granted by the UK National Research Ethics Service Committee - Leeds West (ref. 13/ $\mathrm{YH} / 0349$ ). Thirty-one patients undergoing first-time LA ablation for paroxysmal, persistent, or long-standingpersistent AF were recruited at the Leeds General Infirmary between September 2014 and August 2015, as part of a wider study consecutive cohort $(n=93)$ undergoing biomarker assessment before ablation. Details of the wider cohort have been published $[6,11]$. Of this cohort, 31 participants was the maximum number that could be recruited to the CMR study presented in this article. Patients with systemic inflammatory disease, recent or active malignancy, severe kidney disease (estimated glomerular filtration rate $(\mathrm{eGFR})<30 \mathrm{ml} / \mathrm{min} /$ $1.73 \mathrm{~m}^{2}$ ) connective tissue disease, or any contraindication to CMR were excluded. Written informed consent was gained from all participants.

CMR scans were carried out on a dedicated 1.5 Tesla CMR scanner (Ingenia, Philips Healthcare, Best, Netherlands). Venepuncture was performed immediately prior to the scan, and blood was inserted into potassium EDTA tubes for on-site analysis of haematocrit on a ADVIA 2120 analyser (Siemens Healthineers, Erlangen, Germany). Cine imaging in multiple planes was performed, to allow measurement of standard LA and LV dimensions. Native T1 maps were acquired (electrocardiogram (ECG) triggered $5 \mathrm{~s}(3 \mathrm{~s}) 3 \mathrm{~s}$ Modified Look Locker Inversion Recovery (MOLLI) scheme, reconstructed voxel size $\left.1.2 \times 1.2 \times 10 \mathrm{~mm}^{3}\right)$ on a midventricular short axis slice. Fifteen minutes after administration of $0.15 \mathrm{mmol} / \mathrm{kg}$ intravenous gadolinium based contrast agent a post-contrast T1 map was acquired with identical planning (4s $(2 \mathrm{~s}) 3 \mathrm{~s}(2 \mathrm{~s}) 2 \mathrm{~s}$ MOLLI). ECV was calculated from the pre and post contrast T1 maps [9].

Radiofrequency (RF) ablation was performed according to the 2012 international consensus statement [12]. Under conscious sedation or local anaesthetic, venous access was obtained via the right and left femoral veins. After transseptal puncture, LA bipolar voltages were recorded using a high-density circular electrophysiological (EP) mapping catheter and 3D mapping system (Lasso/CARTO 3, Biosense-Webster or Optima/Ensite Velocity, St. Jude Medical, St. Paul, Minnesota, USA). Mean LA pressures were recorded by transducing the LA sheath. Blood was aspirated from the femoral vein, right atrium, LA, and coronary sinus ostium for later analysis. RF energy was then applied to the PV antra according to standard techniques to perform wide-area circumferential ablation in order to achieve PV isolation. In non-paroxysmal AF, linear ablation or substrate - targeted ablation (e.g. of complex fractionated electrograms) were carried out at the operator's discretion. Successful PV isolation was confirmed in all patients by demonstration of exit and entry block.

Raw EP mapping data were exported from the system according to the manufacturer's instructions, and reformatted to allow 3D geometry and voltage maps to be re-created in analysis software (Paraview). This allowed voltage values to be digitally analysed according to previously published methods [13]; The PV, LA appendage and mitral valve were excluded from analysis. Bipolar voltage of less than $0.5 \mathrm{mV}$ was considered to represent fibrosis, and this was expressed as a percentage of the overall LA endocardial area, excluding the PVs, mitral valve and LA appendage.

Intra-cardiac and peripheral blood aspirated during ablation procedures was analysed using commercially available enzyme-linked immunosorbent assay (ELISA) kits: PIIINP (Elabscience, Beijing, China), gal-3 (Elabscience, Beijing, China) and ICTP (Cusabio Life Science, Wuhan, China). Further details of the ELISA analysis have been previously published [11]. ICTP levels were analysed from coronary sinus blood, gal-3 and PIIINP levels were analysed as a mean of peripheral and intra-cardiac levels, based on the findings of this previous work [11].

All patients were followed up for 365 days according to standard care, with investigation of possible recurrence based on symptoms. In patients without symptoms or documented arrhythmia recurrence after this 365-day period, 24-h electrocardiogram (ECG) monitoring was performed. Arrhythmia recurrence was defined as any documented AF or atrial arrhythmia lasting more than $30 \mathrm{~s}$, occurring more than 60 days after ablation. 


\section{Statistical analysis}

Normally distributed data are expressed as mean \pm standard deviation. Non-parametric data are expressed as median (interquartile range). Categorical data are expressed as frequency (percentage). Data were assessed for normality using the Shapiro-Wilk test and nonparametric data were log-transformed prior to analysis if possible. For comparison, we separated patients into two groups with above and below median LV ECV values. Differences in characteristics between these groups were then assessed using independent-sample t-tests for continuous variables or chi-squared tests for categorical variables. Where transformation of non-parametric data was not possible, Mann-Whitney U test was performed to compare distributions. Univariate linear regression analysis was performed to examine relationships between LV ECV and baseline characteristics. For the multivariable analysis, the forced-entry model was used to identify predictors. Analysis was carried out using SPSS (version 22, Statistical Package for the Social Sciences (SSPS), International Business Machines, Inc., Armonk, New York, USA). A 2-sided $P$-value of $<0.05$ was considered to indicate statistical significance.

\section{Results}

\section{Patient characteristics}

All 31 recruited had CMR assessment. The participants were typical of AF ablation patients and had few comorbidities apart from hypertension (Table 1). Mean LA volume of the cohort was elevated. All patients had LV ejection fraction (LVEF) more than $45 \%$. The majority (80.6\%) had paroxysmal AF (PAF), and the remainder had either persistent or long-standing persistent AF, grouped together for analysis as 'non-PAF'.

\section{Analysis}

Table 2 shows the results of the comparisons between the cohort when split above and below the median LV ECV value of $23.9 \%$. The above-median LV ECV group had higher mean LA pressure $(13 \pm 6 \mathrm{mmHg}$ vs. $8 \pm 4 \mathrm{mmHg}$, $p=0.010)$ and higher ICTP levels (451 (154) ng/ml vs. 212 (146) $\mathrm{ng} / \mathrm{ml}, \quad p=0.001$ ) (Fig. 1). These differences remained significant after multivariable analysis (LAP $\beta=$ 0.791, $p<0.001$ and ICTP $\beta=0.592, p=0.001$ ) (Table 3). The above-median ECV group had a longer duration of AF (52.0 (51.5) months vs 48.8 (57.9) months, $p=0.038$ ), but this was not significant on regression analysis. There was no difference in LA low voltage area between the above and below median ECV groups ( $22 \pm 7 \%$ vs $17 \pm 7 \%$, respectively, $95 \% \mathrm{CI}-1.06$ to $10.9 \% p=0.102)$. No other differences regarding CMR assessment were identified, including the other biomarkers.

In addition to LV ECV, analysis of native T1 mapping values was performed. An association between with ICTP
Table 1 Participant characteristics

\begin{tabular}{|c|c|}
\hline Characteristic & Distribution \\
\hline Age (years) & $56.7 \pm 12.7$ \\
\hline BMI $\left(\mathrm{kg} / \mathrm{m}^{2}\right)$ & $27.5(5.9)$ \\
\hline \multicolumn{2}{|l|}{ Sex } \\
\hline Female & $9(29.0)$ \\
\hline Male & $22(71.0)$ \\
\hline \multicolumn{2}{|l|}{ AF classification } \\
\hline Paroxysmal & 25 (80.6) \\
\hline Non-paroxysmal & $6(19.4)$ \\
\hline Time since AF diagnosis (months) & $51.3(53.9)$ \\
\hline In AF during CMR scan & $9(29.0)$ \\
\hline Diabetes mellitus & $1(3.2)$ \\
\hline Ischaemic heart disease & $2(6.5)$ \\
\hline Hypertension & $9(29.0)$ \\
\hline Systolic blood pressure $(\mathrm{mmHg})$ & $125 \pm 23$ \\
\hline Diastolic Blood pressure (mmHg) & $78 \pm 15$ \\
\hline LA Volume (ml) & $\begin{array}{l}102 \pm 40 \\
\text { minimum 47.5, maximum } 195.4\end{array}$ \\
\hline LV end-diastolic volume (ml) & $158 \pm 37$ \\
\hline LV stroke volume (ml) & $93 \pm 24$ \\
\hline Cardiac output (L/min) & $6.3 \pm 1.6$ \\
\hline LV ejection fraction (\%) & $59.2 \pm 7.1$ \\
\hline LV Mass (g) & $87.3 \pm 24.7$ \\
\hline Native T1 (ms) & $985 \pm 35.5$ \\
\hline LV ECV (\%) & $24.1 \pm 2.5$ \\
\hline Mean LA pressure (mmHg) & $9.0(5.0)$ \\
\hline LA low voltage area (\%) & $20.5 \pm 7.1$ \\
\hline ICTP (ng/ml) & $317.1 \pm 190.1$ \\
\hline Gal-3 (ng/ml) & $36.4 \pm 32.9$ \\
\hline PIIINP (pg/ml) & $76.4 \pm 46.4$ \\
\hline
\end{tabular}

AF Atrial fibrillation, $B M I$ Body mass index, ECV Extracellular volume fraction, gal-3 Galectin 3, ICTP Type I collagen C terminal peptide, LA Left atrial, $L V$ Left ventricular, LVEF Left ventricular ejection fraction, PIIINP Type III procollagen $\mathrm{N}$ terminal peptide

levels was found on univariable analysis (beta $=0.46, p=$ 0.026). Mean LA pressure also approached significance (beta $=0.348, p=0.070$ ). After multivariable analysis, the association with ICTP remained significant (beta $=$ $0.44, p=0.043$ ) but the association with mean LA pressure did not.

\section{Discussion}

\section{Associations with CMR T1 mapping parameters}

Recent studies have demonstrated that T1 mapping during AF is not only feasible but can give important clinical information $[14,15]$. In this study, the presence of AF during the scan appeared to have no effect on the ECV values. 
Table 2 LV ECV comparison

\begin{tabular}{|c|c|c|c|}
\hline & \multicolumn{3}{|l|}{ LV ECV } \\
\hline & Above median LV ECV & Below median LV ECV & $P$ Value \\
\hline ICTP ng/ml & $451(154)$ & $212(146)$ & 0.001 \\
\hline Gal-3 ng/ml & $29.2(19.4)$ & $43.5(41.8)$ & 0.241 \\
\hline PIIINP pg/ml & $63.4(50.4)$ & $87.2(58.0)$ & 0.413 \\
\hline LA voltage $(\%<0.5 \mathrm{mV})$ & $22.3 \pm 7.0$ & $17.4 \pm 6.6$ & 0.102 \\
\hline Mean LA pressure (mmHg) & $13 \pm 6$ & $8 \pm 4$ & 0.010 \\
\hline Age (years) & $56.5 \pm 13.0$ & $56.9 \pm 12.9$ & 0.918 \\
\hline Time since AF diagnosis (months) & $52.0(31.5)$ & $48.8(57.9)$ & 0.038 \\
\hline BMI $\left(\mathrm{kg} / \mathrm{m}^{2}\right)$ & $27.4 \pm 4.3$ & $29.3 \pm 6.0$ & 0.316 \\
\hline LA vol/BSA $\left(\mathrm{ml} / \mathrm{m}^{2}\right)$ & $46.6(21.5)$ & $42.9(15.9)$ & 0.740 \\
\hline LV EDV/BSA $\left(\mathrm{ml} / \mathrm{m}^{2}\right)$ & $71.4 \pm 14.4$ & $79.3 \pm 14.9$ & 0.150 \\
\hline LV ejection fraction (\%) & $61 \pm 8$ & $57 \pm 6$ & 0.140 \\
\hline LV Mass/BSA (g/m²) & $43.3(8.7)$ & $39.1(10.1)$ & 0.522 \\
\hline Systolic blood pressure (mmHg) & $122.8 \pm 21.3$ & $127.4 \pm 25.8$ & 0.590 \\
\hline Diastolic blood pressure (mmHg) & $78.9 \pm 12.1$ & $76.3 \pm 17.0$ & 0.616 \\
\hline PAF & $13(86.7)$ & $12(80.0)$ & 0.930 \\
\hline Male & $10(66.7)$ & $12(80.0)$ & 0.283 \\
\hline Sinus rhythm during scan & $11(73.3)$ & $11(73.3)$ & 1.00 \\
\hline Recurrence of AF & $8(53.3)$ & $7(46.7)$ & 0.715 \\
\hline
\end{tabular}

The median value for LV ECV is $24.0 \%$. Values are 'mean \pm standard deviation', 'median (interquartile range)' or 'frequency (\%)'. $P$ values represent results of Student's t-test for normally distributed data, Mann-Whitney U test for non-parametric data, and chi-squared test for categorical data. Statistically significant results highlighted in bold. BSA Body surface area, EDV End-diastolic volume, PAF Paroxysmal atrial fibrillation
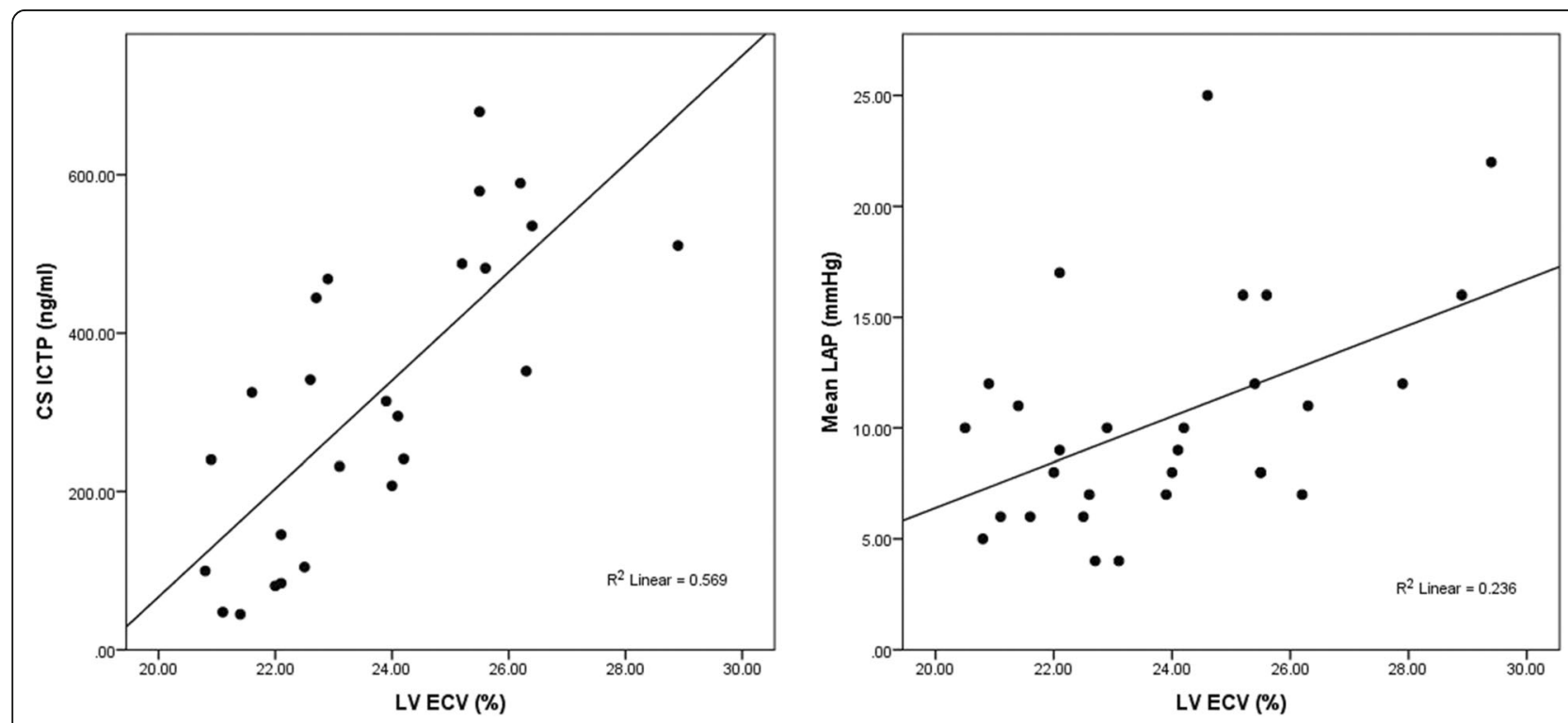

Fig. 1 Scatterplots of associations between type I collagen C terminal telopeptide (ICTP)/left ventricular (LV) extracellular volume fraction (ECV) and left atrial (LA) pressure/LV ECV 
Table 3 Regression analysis

\begin{tabular}{lll}
\hline Characteristic & \multicolumn{2}{l}{ Association with ECV } \\
\cline { 2 - 3 } & Beta & P Value \\
\hline Age (years) & -0.172 & 0.354 \\
Time since AF diagnosis (months) & 0.038 & 0.849 \\
BMI (kg/m ${ }^{2}$ ) & -0.267 & 0.146 \\
Female sex & 0.296 & 0.106 \\
Non - PAF & 0.116 & 0.535 \\
Hypertension & 0.103 & 0.582 \\
CHA ${ }_{2}$ DS 2 VASc & 0.008 & 0.964 \\
LA vol (ml) & -0.247 & 0.181 \\
LV EDV (ml) & -0.294 & 0.115 \\
LV ejection fraction (\%) & 0.184 & 0.330 \\
LV Mass (g) & -0.250 & 0.183 \\
Systolic blood pressure (mmHg) & 0.033 & 0.859 \\
Diastolic blood pressure (mmHg) & 0.069 & 0.714 \\
Mean LA pressure (mmHg) & 0.486 & 0.008 \\
\% LA voltage (<0.5 mV) & 0.791 & $<0.001$ \\
ICTP & 0.257 & 0.225 \\
PIIINP & 0.754 & $<0.001$ \\
Gal-3 & 0.592 & $\mathbf{0 . 0 0 1}$ \\
\hline
\end{tabular}

Results in italics represent multivariable analysis results. Results in bold represent statistically significant associations after multivariable analysis

We have shown that mean LA pressure is associated with LV ECV in AF patients, to our knowledge a novel finding.

LV fibrosis appears to be more pronounced in AF patients than in non-AF controls [16]. A potential mechanistic explanation for this is that LV end-diastolic pressure is elevated in the presence of increased ventricular stiffness and diastolic dysfunction, and this in turn causes an increase in LA pressure, dimension and altered function as a result of the increased atrial workload during ventricular diastole [17]. In their analysis of over 400 patients, Park et al showed that elevated LA pressure is associated with both electro-anatomical remodelling of the LA, and AF recurrence after ablation [10]. It therefore follows that an increase in LV ECV may be related to an increase in LA pressure as seen in our study, and, speculatively, incidence and prognosis of AF.

An association between increasing duration of $\mathrm{AF}$ and $\mathrm{ECV}$, and in keeping with this, between persistent $\mathrm{AF}$ and increased ECV, would be expected based on previous research [7]. Although there was a higher duration of AF in the above-median ECV group, this association was not shown to be significant after regression analysis. An explanation for this may be that the study by Neilan et. al, [7], demonstrating the predictive value of LV ECV for AF recurrence, was much larger $(n=145)$ and better powered to detect subtle associations.
Most LV ECV values recorded in this study were within the normal range; indeed, in comparison with data published from our centre, ECV is equivalent to sedentary healthy controls and lower than ECV derived from cohorts with established myocardial pathology [18-20]. This is likely to be due to the patient group selected for this study; those patients undergoing AF ablation are generally at an early point in the development of their AF, predominantly in paroxysmal rather than persistent or long-standing persistent AF, and have little or no clinically relevant underlying structural heart abnormality. This technique may be able to identify at an early stage in the disease process those patients with a lower chance of rhythm control success when AF has been diagnosed. At least one previous study has suggested this, and further research is required to explore this concept further [7].

The other association with LV ECV and native T1 described in this study is with ICTP levels. To our knowledge, this is a novel finding in both instances. ICTP is a product of the catabolism of type 1 collagen, the most abundant form collagen in the myocardium. Studies examining its predictive value in $\mathrm{AF}$ ablation are sparse and heterogeneous, but there has been some suggestion that it predicts AF recurrence after rhythm control intervention [21, 22]. In previous work we have shown that coronary sinus ICTP levels are higher than intra-atrial levels in this AF patient cohort, suggesting that the predominant site of increased type-I collagen turnover is the ventricle [11]. This should be considered when interpreting studies which have examined circulating ICTP levels in the context of AF - the association between ICTP and AF may represent ventricular pathology, not atrial $[11,23$, 24]. This association may warrant further study, particularly to ascertain any clinical benefit of using this biomarker in AF recurrence risk stratification, or the identification of patients who may benefit from more extensive LA ablation than pulmonary vein isolation.

\section{Association with LA low voltage}

LA voltage mapping data was used as a surrogate marker of LA fibrosis. Although there was more LA low-voltage area in the above-median ECV group, this difference was not significant. Other studies have found low voltage tissue in the LA to be an independent predictor of AF recurrence $[5,6]$. The reason for this discrepancy is not clear, but may be related to the small sample size of this study.

\section{Limitations}

A main limitation of this study is the small number of participants. Nevertheless, the study population is representative of AF ablation patients in general and the multiple modality assessment of fibrosis, coupled with the 
measurement of LA pressure, is unique and has provided novel insights.

A clearly defined value for 'fibrotic' tissue based on histological validation was not used, however the results (particularly the association between ICTP and LV ECV) do imply such a relationship exists. In this study, there was no control group with which to compare ECV values. It should be noted that isolated measurement of LA pressure during an ablation procedure may not reflect chronic load status, however repeated or continuous direct LA pressure monitoring is not feasible and the size of this potential error is unknown.

\section{Conclusion}

Higher LV ECV in AF patients is associated with higher LA pressure and type 1 collagen turnover.

\begin{abstract}
Abbreviations
AF: Atrial fibrillation; BMI: Body mass index; BSA: Body surface area; CMR: Cardiovascular magnetic resonance; ECG: Electrocardiogram; ECV: Extracellular volume fraction; EDV: End-diastolic volume; eGFR: Estimated glomerular filtration rate; EP: Electrophysiology; Gal-3: Galectin 3; ICTP: Type I collagen C terminal peptide; LA: Left atrium/left atrial; LV: Left ventricle/left ventricular; LVEF: Left ventricular ejection fraction; MOLLI: Modified Look Locker inversion recovery; PAF: Paroxysmal atrial fibrillation; PIIINP: Type III procollagen $\mathrm{N}$ terminal peptide; PV: Pulmonary vein; RF: Radiofrequency
\end{abstract}

\section{Acknowledgements}

The authors wish to thank all the cardiac electrophysiology consultants, as well as the nursing, radiography and cardiac physiology staff of the cardiac catheter laboratories, CMR department, and Cardiovascular Clinical Research Facility at the Leeds General Infirmary.

\section{Authors' contributions}

GAB: Project conception, all patient contact and interventions, supervision of CMR scans, data acquisition and analysis, drafting and finalisation of manuscript. PPS: Project conception, implementation of CMR protocol, supervision of CMR scans, data acquisition and analysis, review of manuscript. RK: Development of CMR protocol, analysis of raw left atrial electrophysiological mapping data, review of manuscript. TO: Analysis of raw left atrial electrophysiological mapping data, review of manuscript. KR: Development of CMR protocol, review of manuscript. AVH: Project conception, data analysis, review of manuscript. JPG: Provision of CMR facilities, review of manuscript. ES: Project conception, review of manuscript. GYHL: Project conception, provision of laboratory facilities for ELISA, review of manuscript. SP: Project conception, provision of CMR facilities, review of manuscript. MHT: Project conception, review of manuscript. All authors read and approved the final manuscript.

\section{Funding}

Rashed Karim was supported by the National Institute for Health Research Biomedical Research Centre at Guy's and St. Thomas' NHS Foundation Trust and King's College London. Sven Plein is funded by British Heart Foundation fellowships (FS/1062/28409). The work of Tobias Oesterlein is funded by the German Research Foundation under grant D0637/14-1. Additional finding was from the LGI cardiac electrophysiology research fund. The study was supported by the National Institute for Health Research, Leeds Clinical Research Facility. The views expressed are those of the authors and not necessarily those of the NHS, NIHR or the Department of Health.

\section{Availability of data and materials}

The dataset used during the current study is available from the corresponding author on reasonable request.

\section{Ethics approval and consent to participate}

The study was approved by the National Research Ethics Service $(13 / \mathrm{YH} /$ 0349). Written informed consent was obtained from all participants.

\section{Consent for publication}

Not applicable.

\section{Competing interests}

St. Jude Medical supported Gordon Begg's research fellowship. Gregory Lip has served as a consultant for Bayer/Jansen, BMS/Pfizer, Biotronik, Medtronic, Boehringer Ingelheim, Microlife, and Daiichi-Sankyo, and has been a speaker for Bayer/Jansen, BMS/Pfizer, Medtronic, Boehringer Ingelheim, Microlife, Roche and Daiichi-Sankyo. Muzahir Tayebjee has received research grants from St. Jude Medical, Medtronic, Biosense Webster, and Boehringher Ingelheim. Dr. Dana Peters served as the JCMR Guest Editor for this manuscript.

\section{Author details}

${ }^{1}$ Department of Cardiology, Leeds General Infirmary, X39 Cardiology Offices, Great George St, Leeds LS1 3EX, UK. '²eeds Institute of Cardiovascular and Metabolic Medicine, University of Leeds, Clarendon Way, Leeds LS2 9JT, UK. ${ }^{3}$ Department of Biomedical Engineering, King's College, London, UK. ${ }^{4}$ Institute of Biomedical Engineering, Karlsruhe Institute of Technology (KIT), 76131 Karlsruhe, Germany. ${ }^{5}$ MCRC and School of Biomedical Sciences, University of Leeds, Leeds LS2 9JT, UK. 'University of Birmingham Institute of Cardiovascular Sciences, City Hospital, Birmingham, UK. ${ }^{7}$ University of Liverpool, Liverpool, UK. ${ }^{8}$ Aalborg Thrombosis Research Unit, Department of Clinical Medicine, Aalborg University, Aalborg, Denmark.

Received: 11 March 2019 Accepted: 15 January 2020

Published online: 10 February 2020

\section{References}

1. Weerasooriya R, Khairy P, Litalien J, Macle L, Hocini M, Sacher F, Lellouche N, Knecht S, Wright M, Nault I, et al. Catheter ablation for atrial fibrillation: are results maintained at 5 years of follow-up? J Am Coll Cardiol. 2011;57:160-6.

2. Begg GA, Holden AV, Lip GY, Plein S, Tayebjee MH. Assessment of atrial fibrosis for the rhythm control of atrial fibrillation. Int J Cardiol. 2016;220:155-61.

3. Goette A, Kalman JM, Aguinaga L, et al. EHRA/HRS/APHRS/SOLAECE expert consensus on Atrial cardiomyopathies: Definition, characterisation, and clinical implication. J Arrhythm. 2016;32(4):247-78. https://doi.org/10.1016/j. joa.2016.05.002.

4. Marrouche NF, Wilber D, Hindricks G, Jais P, Akoum N, Marchlinski F, Kholmovski E, Burgon N, Hu N, Mont L, et al. Association of atrial tissue fibrosis identified by delayed enhancement MRI and atrial fibrillation catheter ablation: the DECAAF study. JAMA. 2014;311:498-506.

5. Verma A, Wazni OM, Marrouche NF, Martin DO, Kilicaslan F, Minor S, Schweikert RA, Saliba W, Cummings J, Burkhardt JD, et al. Pre-existent left atrial scarring in patients undergoing pulmonary vein antrum isolation: an independent predictor of procedural failure. J Am Coll Cardiol. 2005;45:285-92.

6. Begg GA, Karim R, Oesterlein T, Graham LN, Hogarth AJ, Page SP, Pepper CB, Rhode K, Lip GYH, Holden AV, et al. Left atrial voltage, circulating biomarkers of fibrosis, and atrial fibrillation ablation. A prospective cohort study. PLoS One. 2018;13:e0189936.

7. Neilan TG, Mongeon FP, Shah RV, Coelho-Filho O, Abbasi SA, Dodson JA, McMullan CJ, Heydari B, Michaud GF, John RM, et al. Myocardial extracellular volume expansion and the risk of recurrent atrial fibrillation after pulmonary vein isolation. JACC Cardiovasc Imaging. 2014;7:1-11.

8. Shantsila E, Shantsila A, Blann AD, Lip GY. Left ventricular fibrosis in atrial fibrillation. Am J Cardiol. 2013;111:996-1001.

9. Moon JC, Messroghli DR, Kellman P, Piechnik SK, Robson MD, Ugander M, Gatehouse PD, Arai AE, Friedrich MG, Neubauer S, et al. Myocardial T1 mapping and extracellular volume quantification: a Society for Cardiovascular Magnetic Resonance (SCMR) and CMR Working Group of the European Society of Cardiology consensus statement. J Cardiovasc Magn Reson. 2013;15:92.

10. Park J, Joung B, Uhm JS, Young Shim C, Hwang C, Hyoung Lee M, Pak HN. High left atrial pressures are associated with advanced electroanatomical remodeling of left atrium and independent predictors for clinical recurrence of atrial fibrillation after catheter ablation. Heart Rhythm. 2014;11:953-60.

11. Begg GA, Karim R, Oesterlein T, Graham LN, Hogarth AJ, Page SP, Pepper CB, Rhode K, Lip GYH, Holden AV, Plein S, Tayebjee MH. Intra-cardiac and peripheral levels of biochemical markers of fibrosis in patients undergoing 
catheter ablation for atrial fibrillation. Europace. 2017;19(12):1944-1950. https://doi.org/10.1093/europace/euw315.

12. Calkins H, Kuck KH, Cappato R, Brugada J, Camm AJ, Chen SA, Crijns HJ, Damiano RJ Jr, Davies DW, DiMarco J, et al. 2012 HRS/EHRAVECAS expert consensus statement on catheter and surgical ablation of atrial fibrillation: recommendations for patient selection, procedural techniques, patient management and follow-up, definitions, endpoints, and research trial design: a report of the Heart Rhythm Society (HRS) Task Force on Catheter and Surgical Ablation of Atrial Fibrillation. Developed in partnership with the European Heart Rhythm Association (EHRA), a registered branch of the European Society of Cardiology (ESC) and the European Cardiac Arhythmia Society (ECAS); and in collaboration with the American College of Cardiology (ACC), American Heart Association (AHA), the Asia Pacific Heart Rhythm Society (APHRS), and the Society of Thoracic Surgeons (STS). Endorsed by the governing bodies of the American College of Cardiology Foundation, the American Heart Association, the European Cardiac Arrhythmia Society, the European Heart Rhythm Association, the Society of Thoracic Surgeons, the Asia Pacific Heart Rhythm Society, and the Heart Rhythm Society. Heart Rhythm. 2012;9:632-696.e621.

13. Oesterlein TG, Schmid J, Bauer S, Jadidi A, Schmitt C, Dössel O, Luik A. Analysis and visualization of intracardiac electrograms in diagnosis and research: concept and application of KaPAVIE. Comput Methods Prog Biomed. 2016;127:165-73.

14. Zhao L, Li S, Ma X, Bai R, Liu N, Li N, Schoenhagen P, Ma C. Prognostic significance of left ventricular fibrosis assessed by $\mathrm{T} 1$ mapping in patients with atrial fibrillation and heart failure. Sci Rep. 2019:9:13374.

15. Zhao L, Li S, Ma X, Greiser A, Zhang T, An J, Bai R, Dong J, Fan Z. Systolic MOLLI T1 mapping with heart-rate-dependent pulse sequence sampling scheme is feasible in patients with atrial fibrillation. J Cardiovasc Magn Reson. 2016;18:13.

16. Ling LH, Kistler PM, Ellims AH, lles LM, Lee G, Hughes GL, Kalman JM, Kaye DM, Taylor AJ. Diffuse ventricular fibrosis in atrial fibrillation: noninvasive evaluation and relationships with aging and systolic dysfunction. J Am Coll Cardiol. 2012;60:2402-8.

17. Mitchell JH, Gilmore JP, Sarnoff SJ. The transport function of the atrium. Factors influencing the relation between mean left atrial pressure and left ventricular end diastolic pressure. Am J Cardiol. 1962;9:237-47.

18. Swoboda PP, McDiarmid AK, Erhayiem B, Broadbent DA, Dobson LE, Garg P, Ferguson C, Page SP, Greenwood JP, Plein S. Assessing myocardial extracellular volume by T1 mapping to distinguish hypertrophic cardiomyopathy from athlete's heart. J Am Coll Cardiol. 2016;67:2189-90

19. Garg P, Broadbent DA, Swoboda PP, Foley JRJ, Fent GJ, Musa TA, Ripley DP, Erhayiem B, Dobson LE, McDiarmid AK, et al. Acute infarct extracellular volume mapping to quantify myocardial area at risk and chronic infarct size on cardiovascular magnetic resonance imaging. Circ Cardiovasc Imaging. 2017;10. https://doi.org/10.1161/CIRCIMAGING.117.006182

20. Fent GJ, Garg P, Foley JRJ, Swoboda PP, Dobson LE, Erhayiem B, Treibel TA, Moon JC, Greenwood JP, Plein S. Synthetic myocardial extracellular volume fraction. JACC Cardiovasc Imaging. 2017:10:1402-4.

21. Richter B, Gwechenberger M, Socas A, Zorn G, Albinni S, Marx M, Wolf F, Bergler-Klein J, Loewe C, Bieglmayer C, et al. Time course of markers of tissue repair after ablation of atrial fibrillation and their relation to left atrial structural changes and clinical ablation outcome. Int J Cardiol. 2011;152:231-6.

22. Kimura T, Takatsuki S, Inagawa K, Katsumata Y, Nishiyama T, Nishiyama N, Fukumoto K, Aizawa Y, Tanimoto Y, Tanimoto K, Fukuda K. Serum inflammation markers predicting successful initial catheter ablation for atrial fibrillation. Heart Lung Circ. 2014;23:636-43.

23. Fujita $M$, Cheng XW, Inden $Y$, Shimano M, Yoshida N, Inoue A, Yamamoto T, Takeshita K, Kyo S, Taguchi N, et al. Mechanisms with clinical implications for atrial fibrillation-associated remodeling: cathepsin $\mathrm{K}$ expression, regulation, and therapeutic target and biomarker. J Am Heart Assoc. 2013;2:e000503.

24. Okumura Y, Watanabe I, Nakai T, Ohkubo K, Kofune T, Kofune M, Nagashima K, Mano H, Sonoda K, Kasamaki Y, Hirayama A. Impact of biomarkers of inflammation and extracellular matrix turnover on the outcome of atrial fibrillation ablation: importance of matrix metalloproteinase-2 as a predictor of atrial fibrillation recurrence. J Cardiovasc Electrophysiol. 2011;22:987-93.

\section{Publisher's Note}

Springer Nature remains neutral with regard to jurisdictional claims in published maps and institutional affiliations.

\section{Ready to submit your research? Choose BMC and benefit from:}

- fast, convenient online submission

- thorough peer review by experienced researchers in your field

- rapid publication on acceptance

- support for research data, including large and complex data types

- gold Open Access which fosters wider collaboration and increased citations

- maximum visibility for your research: over $100 \mathrm{M}$ website views per year

At BMC, research is always in progress.

Learn more biomedcentral.com/submissions 